\title{
Dental students' ability to locate emergency equipment- lessons learned from aviation
}

\author{
\begin{tabular}{l|l|l} 
H. M. Pinsky & | J. M. Le & | D. G. Sweier \\
& | K. Marti
\end{tabular}
}

${ }^{1}$ Department of Periodontics \& Oral Medicine, University of Michigan School of Dentistry, Ann Arbor, MI, USA

${ }^{2}$ University of Michigan School of Dentistry, Ann Arbor, MI, USA

${ }^{3}$ Department of Cariology, Restorative Sciences \& Endodontics, University of Michigan School of Dentistry, Ann Arbor, MI, USA

${ }^{4}$ Department of Oral and Maxillofacial Surgery/Hospital Dentistry, University of Michigan School of Dentistry, Ann Arbor, MI, USA

\section{Correspondence}

Harold M. Pinsky, DDS, Department of Periodontics \& Oral Medicine, University of Michigan School of Dentistry, Ann Arbor, MI, USA.

Email: hmpinsky@umich.edu

Funding information

This study was supported by the Leadership Pathway Program, University of Michigan School of Dentistry.

\begin{abstract}
Purpose: The purpose of this study was to evaluate the dental student's ability to locate medical emergency equipment/items at the University of Michigan School of Dentistry clinic.

Methods: A total of 138 second-year dental students (traditional group) participated in this study as part of a simulation-based medical emergencies rotation course held during the winter term of 2014 and 2015. Without prior training, students were tested on their ability to locate nine predetermined items on the clinic floor using a selfreported checklist. Six months later, a convenience sample of 18 students (novel group) from the same cohort were later trained on their location and retested individually.

Results: Of the 138 students tested, only $10.14 \%$ students could locate seven of the nine items when compared to $100 \%$ in the novel group. Only $5.07 \%$ of students in the traditional group could locate all items initially, compared with $72.22 \%$ students in the novel group.

Conclusion: Whilst our students have lecture-based knowledge about medical emergencies, the results of our study identified a gap of knowledge of emergency equipment/item location amongst students. Therefore, an intervention performed with a similar group of second-year dental students supported that proper training may be used to achieve retention of knowledge. Based on our "novel group" results, we have incorporated targeted training in the dental curriculum that leads to students being better prepared in locating emergency equipment/items. This study suggests that other populations, such as faculty or staff, may also benefit from hands-on training.
\end{abstract}

KEYWORDS

training, dentistry, emergencies, simulation, emergency equipment, undergraduate dental education

\section{1 | INTRODUCTION}

Patient safety is at the very foundation of comprehensive dental care. A necessary part of dental care is the need to be able to manage medical emergencies when they arise. Traditional training in managing medical emergencies in the dental school begins with lecture-based coursework. ${ }^{1}$ Training then continues with evidence-based instruction on the theory of managing specific emergency situations towards active learning educational methods. ${ }^{2-4}$ Teaching safety in emergency management can be divided into three building blocks: (i) the location of emergency equipment, (ii) the operation of emergency equipment and (iii) the understanding of when and how to properly use a particular piece of equipment. To accomplish these three components effectively, proper training of equipment location and operation is best accomplished in an environment that simulates the experience. At the University of Michigan School of Dentistry (UMSoD), students 
are exposed to medical emergency training once, in lecture format and role-playing simulation rotation and recurrent training is non-existent and not mandated. Typical lecture-based learning is inefficient to practice emergencies, as demonstrated in other fields such as aviation, first responders and others, who are constantly honing their skills. ${ }^{5-7} \mathrm{We}$ identified a gap; at no point in the curriculum were our students taken to the physical location of the medical equipment in the School of Dentistry.

Recently, dentistry has looked to the airline industry and their Crew Resource Management (CRM) for guidance on medical emergencies management. ${ }^{8}$ CRM is defined as a management system which optimises use of all resources, specifically equipment, information, and people, to promote safety and efficiency. One component of CRM is to have properly located equipment and properly trained personnel to use that equipment. In dentistry, the location of emergency equipment and the ability of the students to use them effectively are crucial in managing a medical emergency. The translation of knowledge of emergency treatment to practical application mandates knowledge of emergency equipment location and correct use. ${ }^{9}$ Furthermore, it is not unrealistic to expect every personnel on a health team, including faculty, staff and students, to be able to find this equipment in their clinical environment. Specific to managing an aviation emergency, it is well known that the airline industry tends to operate multiple fleets and model types with the potential for a significant difference in interior arrangement and design. Although the interior design of each aircraft model type is unique, flight attendants' training includes operating and managing an emergency in the cabin, based on the standardised location of specific emergency equipment. Consequently, during a simulated emergency training session, whilst there may be a multitude of possible medical emergency scenarios, it is essential to keep the medical supplies and equipment predictably in a consistent location and space so that a well-trained individual can find it. ${ }^{10}$

According to the Committee on Dental Accreditation (CODA), it is an expectation for the graduating dental student in the United States to "be able to manage common medical emergencies." ${ }^{11}$ At the same time, when planning to provide pre-clinical medical emergency management training in the dental curriculum, it remains absolutely necessary that these skills be quantitatively assessed for outcomes. ${ }^{12,13}$ We initiated a newly developed simulation-based medical emergencies course for second-year dental students based on Kolb's theory of experiential learning. This course included a quantitative assessment of students' training in medical emergencies recognition and management. Kolb's theory shows that effective learning is accomplished when a person progresses through a cycle of four stages: (i) concrete experience, (ii) reflective observation, (iii) abstract conceptualisation and (iv) active experimentation. This is achieved as the learner first has the actual experience, then is given the opportunity to reflect on the experience, learns from the experience and eventually has the opportunity to try out (repeat) the experience again. ${ }^{10}$

Interestingly, this teaching method is being utilised for dental procedures, but is underemphasised regarding emergency training. Furthermore, there has been no attempt to report on the use of medical equipment location in dental schools. Therefore, we aim to alleviate this important deficit by studying the effect of a innovative training program for dental students on their ability to locate emergency equipment in dental clinics. The purpose of this study was to determine whether an innovative hands-on training program was effective in supporting dental students as they learned how to locate medical emergency equipment in the clinical setting.

\section{2 | MATERIALS AND METHODS}

This study was conducted as part of the medical emergencies course offered in the winter term of the second year of the dental curriculum (2014 and 2015). This study was reviewed and determined exempt by the University of Michigan Institutional Review Board (IRB) (HUM00086587).

Before the simulation session, all second-year dental students participated in a traditional lecture-based course, including instruction on the use of medical emergency equipment; visual identification of equipment was done using only photographs, see Figure 2, due to the nature of the lecture-based course. Depending on the group, not more than 5 or 6 weeks elapsed between the lecture-based and the simulation-based course. To evaluate the students' ability to locate medical emergency equipment, two senior authors (HMP and KM) in this study generated a list of nine items (Table 1) that were deemed necessary for proper medical emergency management in a dental environment after consulting the relevant literature and based on a previously published dental checklist by Pinsky et al. ${ }^{1,8,14-20}$

Initially, a building floor plan was acquired and the locations of all predetermined items were marked (Figure 1). During the simulation portion of the medical emergencies course, students were asked to locate the list of equipment (Table 1 and Figure 2 ) in the clinic. To document the students' ability to find the items, students were given a list (Table 1) and asked to independently locate them on the thirdfloor clinics. As the "scavenger hunt" was performed during the medical emergencies simulation-based course as previously mentioned, we estimated required time to $10-15$ minutes pre-emptively, and we allowed students to complete their assignment within that timeline. Students were asked to independently mark (i) yes, if they could find

TABLE 1 List of nine medical equipments the students needed to find

\section{Medical equipment/item}

1. Portable oxygen tanks and masks

2. Emergency phone location with information card containing emergency phone numbers

3. Ammonium chloride

4. Red emergency kit

5. Automated external defibrillator (AED)

6. Eye wash

7. Blood pressure cuff and stethoscope, and glucometer

8. Emergency shower

9. Elevator location for emergency medical team/CODE situation 


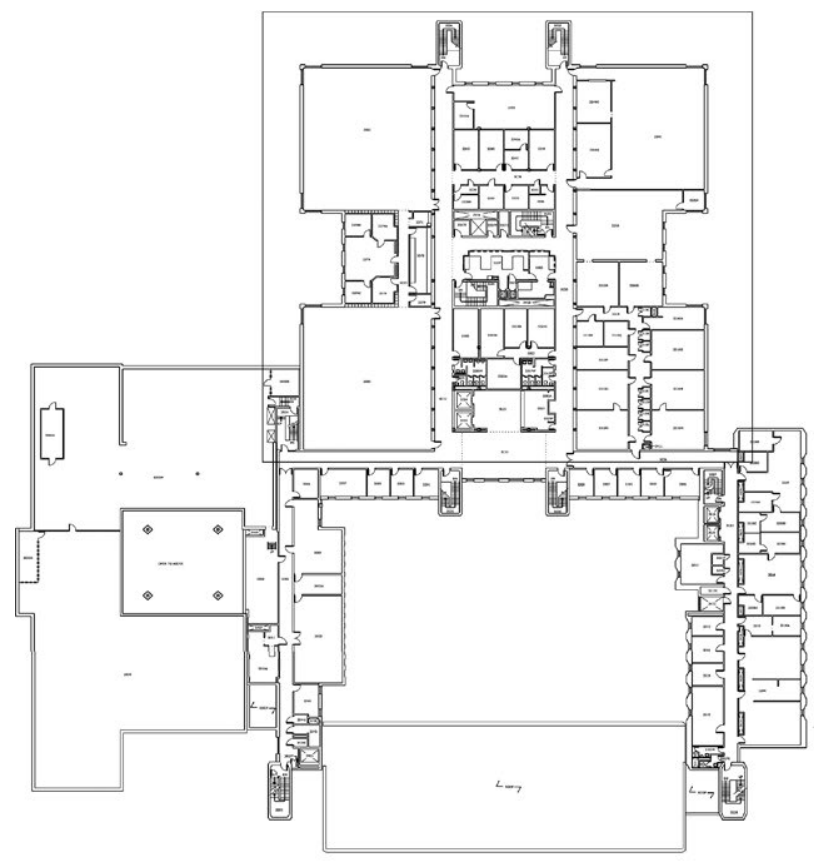

Third Floor Plan

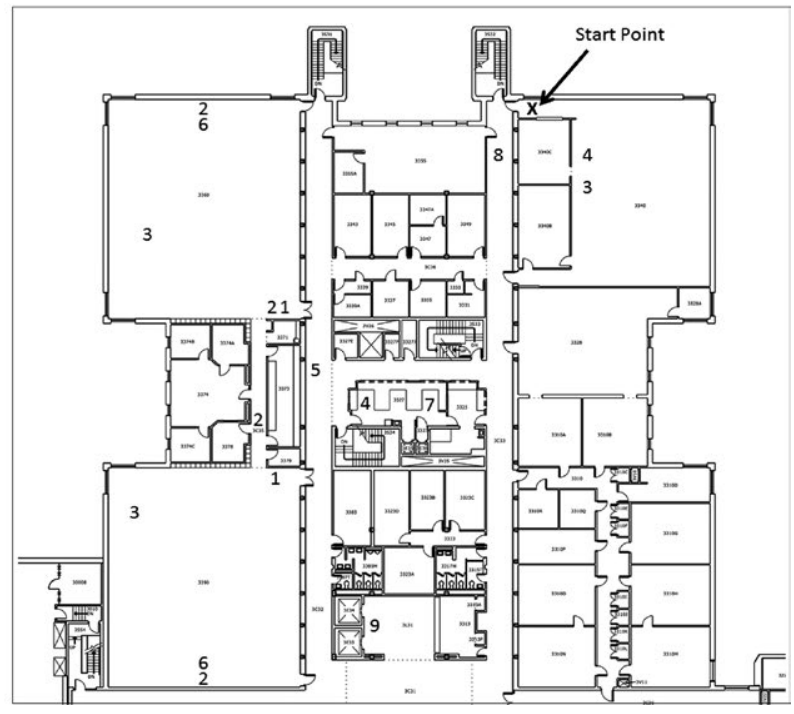

FIGURE 1 Floor layout of the third floor, with start point marked X. The location of each of the listed items is marked on the floor plan. Although the ammonium chloride (item 3 ) is only indicated once in each of the three clinics, it is actually located in each cubicle drawer in the student clinics (35 of cubes per clinic) ${ }^{13}$

the item or (ii) No, if they could not find the item. Students were also informed that no grade would be associated with the assignment. No time limit was imposed on the students. The list was then collected anonymously at the end of the exercise and frequency data were recorded by three of the authors (HMP, KM and DS; Table 2).

Six months later, a convenience group of eighteen students who had participated in the course were arbitrarily sampled by one of the authors (JML) based on availability of the students' time. This "novel group" received additional hands-on training, which consisted of physically locating each item under supervision of an experienced facilitator (JML). This second phase occurred in the same clinical setting used in the initial test. Two weeks later, each student in the "novel group" was assessed individually. This time, each student was followed by the same facilitator who confirmed the ability of the student to locate the equipment and this information was recorded (Table 2). All attempts were made to maximise the student sample in the "novel" group." Due to their limited time and scheduling conflicts, we were not able to increase the number of participants in this group (as presented in Table 2).

\subsection{Analyses}

To evaluate impact of course on the students' ability to successfully locate equipment/items, differences in frequency counts across training groups (traditional and novel) were compared using a chi-square test of independence. $P \leq .05$ was considered statistically significant for two-tailed tests, and frequency rates reported as percentage frequency for both trainee groups.
TABLE 2 Comparison of dental students' ability to find nine items across training programmes using chi-square test of independence

\begin{tabular}{|c|c|c|c|}
\hline Equipment/item & $\begin{array}{l}\text { Traditional } \\
(\mathrm{N}=138) \mathrm{N}(\%)\end{array}$ & $\begin{array}{l}\text { Novel }(n=18) \\
n(\%)\end{array}$ & $P$ value \\
\hline $\begin{array}{l}\text { Portable oxygen tank } \\
\text { and mask }\end{array}$ & $104(75.36)$ & $17(94.44)$ & .059 \\
\hline Emergency phone & $125(90.58)$ & 16 (88.89) & .810 \\
\hline Ammonium chloride & 16 (11.59) & $18(100.00)$ & .001 \\
\hline Red emergency kit & $52(37.68)$ & $17(94.44)$ & .001 \\
\hline $\begin{array}{l}\text { Automated external } \\
\text { defibrillator (AED) }\end{array}$ & $88(63.77)$ & $17(94.44)$ & .009 \\
\hline Eye wash station & $74(53.62)$ & $18(100)$ & .001 \\
\hline $\begin{array}{l}\text { Blood pressure cuff } \\
\text { and stethoscope } \\
\text { and glucometer }\end{array}$ & $88(63.77)$ & $18(100.00)$ & .002 \\
\hline Emergency shower & 89 (64.49) & 16 (88.89) & .038 \\
\hline Emergency elevator & $64(46.38)$ & $18(100.00)$ & .001 \\
\hline
\end{tabular}

\section{3 | RESULTS}

\section{1 | Traditional group}

Of the 138 students tested during the 2014 and 2015 term, the majority could identify the location of the emergency phone (90.58\%) and portable oxygen tank $(75.36 \%)$, whilst a small percentage of students 


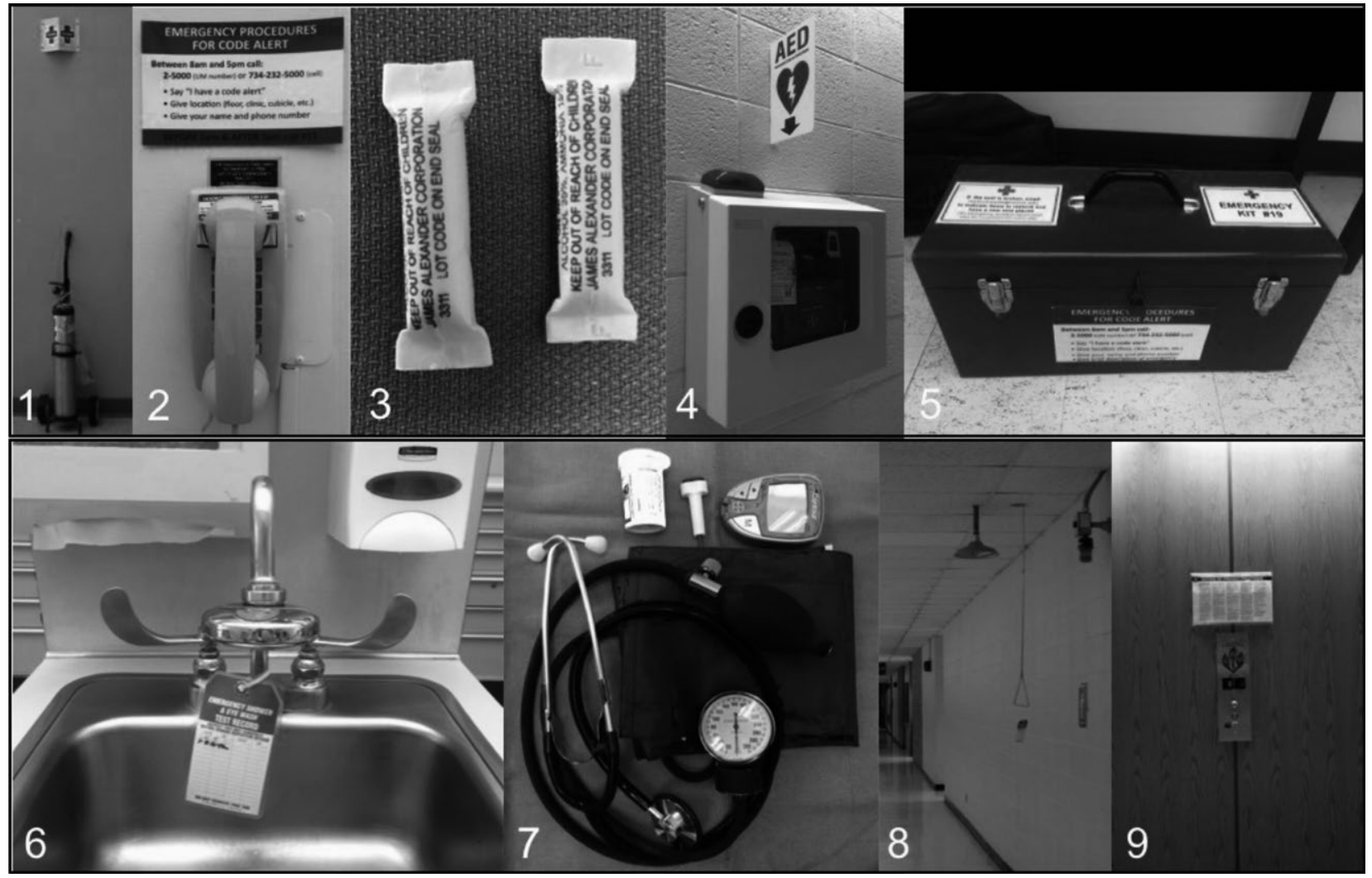

FIGURE 2 Location and signage of the nine items

could locate the ammonium chloride (11.59\%). The remainder of the items included: automated external defibrillator (AED; 63.77\%), red emergency kit (37.6\%), eye wash station (53.62\%), blood pressure and glucometer (63.77\%), emergency shower (64.49\%) and emergency elevator for CODE situation (46.38\%; Table 2). Additionally, $10.14 \%$ of the students could locate seven of the nine items with only $5.07 \%$ students being able to locate all nine items (Figure 3).

\subsection{Novel group $(\mathrm{N}=18)$}

Eighteen ${ }^{18}$ students participated in the novel curriculum that targeted hands-on training. After 2 weeks, the 18 students were tested and $100 \%$ of them could locate seven of the nine following items: oxygen tank, emergency phone, ammonium chloride, red emergency kit, eye wash, blood pressure cuff, stethoscope and glucometer, AED, emergency shower and elevator location for CODE situation. Most the students (94.44\%) could locate the portable oxygen tank and masks, red emergency kit and AED. Finally, the emergency phone and emergency shower had the lowest finding rate (88.89\%; Table 2). Interestingly, all $100 \%$ students could locate at least seven of the nine items, including $72.22 \%$ students who could locate all nine items. (Figure 4).

\subsection{Frequency rates of locating items}

Regardless of training modality, the most commonly found item was emergency phone $(90.58 \%$ and $88.89 \%$, for traditional and novel curricula, respectively), followed by portable oxygen tank and mask (75.36\% and $94.44 \%$ for traditional and novel curricula, respectively).

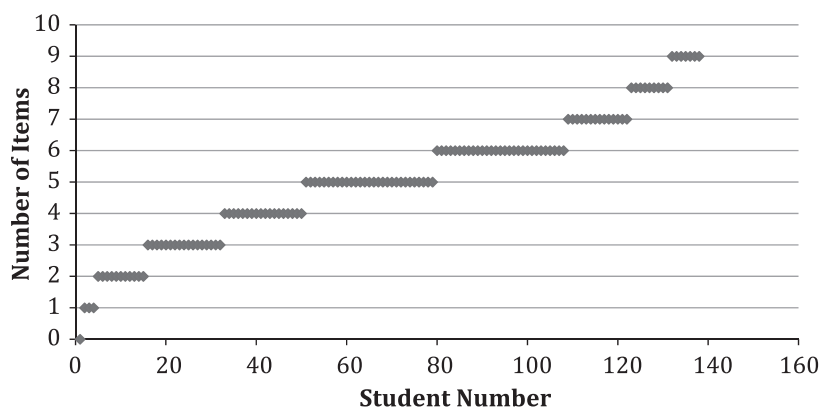

FIGURE 3 Graph of 2014-2015 cohort of 138 students demonstrating each student's ability to find the number of items on the list (rotation groups)

Comparison of frequency rates indicated that for every item in the novel curriculum, where faculty showed the students where each item was located, there was a higher frequency of successfully finding each item. Statistical differences in frequency rates of successfully finding equipment were identified across training modalities for all, but two items - portable oxygen tank and mask and emergency phone (Table 2).

\section{DISCUSSION}

No previous studies have looked at the dental students' ability to locate medical emergency equipment. In a paper discussing availability of emergency equipment, Al-Sebaei et al. ${ }^{21}$ evaluated the preparedness of private dental offices and polyclinics in Western Saudi Arabia and 


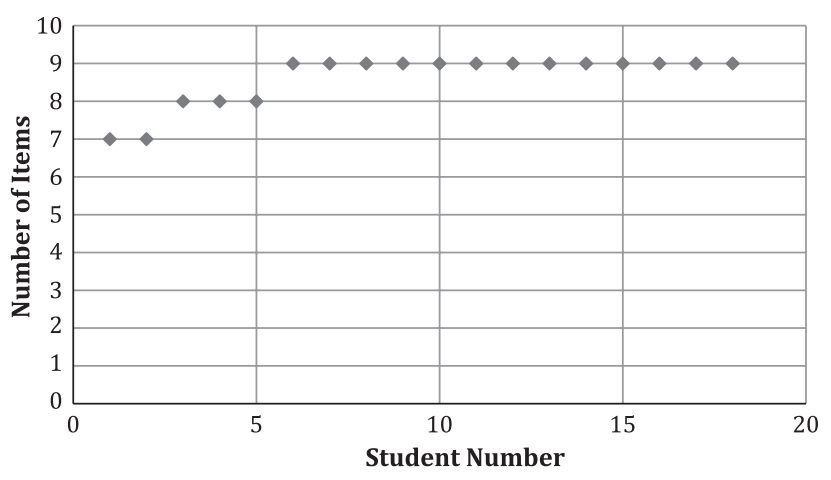

FIGURE 4 Graph of intervention group of 18 students demonstrates each student's ability to find the total number of items on the list (intervention group)

discovered a highly significant deficiency in the availability of emergency drugs and equipment. Although the study reported the mean level of preparedness of dental office personnel at $55 \%$, the availability of drugs and supplies were only $35 \%$ and $19 \%$, respectively. Of the 70 offices surveyed, only seven reported having at least one type of supplemental oxygen delivery device. Additionally, only seven of the 70 offices had an AED and bag-valve mask (BVM). ${ }^{21}$ Moreover, a crosssectional study conducted in 2014 surveyed 250 dental graduates in dental offices in different areas of India and showed that emergency kits were only available at $24 \%$ of the offices. ${ }^{22}$ In summary, there are several documented studies reporting the availability of emergency equipment in dental settings, and a few discoverable studies that surveyed the preparedness of dental office personnel. ${ }^{23-28}$ However, to the authors' knowledge, this current study is the first to directly evaluate dental students' ability to physically locate emergency equipment in their dental school clinics.

In dental schools, instruction on the use of emergency equipment in lecture formats has been well documented. ${ }^{18,29,30}$ However, as part of one study at the same institution, Le et al. ${ }^{13}$ reported the ability of the students to locate portable oxygen tanks in simulated exercises. In that study, Le et al. found that only $68 \%$ of UMSoD third- and fourthyear dental students could correctly locate oxygen tanks when asked to do so as part of a simulated medical emergency. After the Le et al. study, the ability of students to locate the portable oxygen tanks remains $75 \%$ in our study, despite the initiation of clear identification signage of the position of the oxygen in the clinical setting, initiated because of the Le et al. paper.

The UMSoD curriculum is replete with examples of instruction and skill acquisition using the Miller's pyramid: "knows, knows how, shows how and does." ${ }^{31}$ Examples include technical dental procedures such as fabrication of dentures or tooth preparation for a crown. First, the students are provided didactic instruction; they then practice the skills in a simulated preclinical setting; and only then do they provide the treatment in supervised patient care. ${ }^{2}$ Similarly, the ability to successfully locate emergency medical equipment is an acquired skill. This skill had previously only been taught using didactic instruction, the "tell" part of "tell, show and do."32 Using a "scavenger hunt" methodology, this study demonstrated a gap in the knowledge and that only using "knows" is not sufficient, we have interpreted the "knows how" part of the pyramid, as the "of being functionally adequate, or of having sufficient knowledge, judgement, skill or strength for a particular duty" as described by Miller. ${ }^{31}$ By incorporating the "shows how" and "does," we clearly demonstrated that students could correctly locate medical emergency equipment after hands-on training. ${ }^{33}$

The present study demonstrates that hands-on training on the physical location of medical emergency equipment is essential. Because of this study, a modification in the UMSoD curriculum has been approved. A module has been added to the simulated medical emergency course for second-year dental students. This module specifically provides hands-on instruction on the location of medical emergency equipment to correct the gap in knowledge that we have identified.

There are limitations to consider in this study. First, the initial study data were self-reported leaving the possibility of students overreporting, "yes" marks. But, examination of data showed that only two students reported finding all nine items prior to any training. Therefore, if over-reporting were true, then it only occurred in a very small percentage of the sample (Figure 3). Second, even though the students were instructed by the facilitators to complete the "scavenger hunt" independently without consulting other colleagues and staff on the clinic floor, they were not individually monitored during this exercise. Our study design did not have a method to restrict students from communicating with each other during the session. Additionally, the scavenger hunt was broken into multiple sessions on different dates. Thus, students who had completed their session may have had communicated their experiences to colleagues who had not experienced the exercise. Third, the "novel" group was a subset sample selected based on availability and further efforts to increase their number was not feasible due to time constraints even though our original plan was to test all students. Fourth was that we only tested 18 students at two or more weeks for knowledge retention of medical equipment location, and the resultant statistical analysis should be viewed with that in mind.

We have initiated mandatory hands-on training on all items. A dedicated physical tour of the facility emphasising the actual location of medical emergency equipment has been instituted for the following year. Based on our findings, and the subsequent preparation for Accreditation in our school, a complete building standardisation of location of emergency equipment was accomplished. Furthermore, we are also considering implementing this recurrent training on an annual basis like the airline pilot best practices. The literature, although limited to nursing and to skills associated with Advanced Cardiac Life Support (ACLS) training, supports a recurrent training 6 months and 1 year after initial training. ${ }^{34,35}$

Like the mandated recurrent training required by the Federal Aviation Administration (FAA) for airline pilots, our plan is to institute refresher training for dental students every 6 months to 1 year. For acquisition of long-term data, we propose further reassessment of students at least 2-3 years' post-intervention. With the knowledge provided through this training, the students will be better prepared to locate medical emergency equipment. Due to this newly identified gap, we are expanding our findings to include training for dental hygiene students. Additional options for improvement in students' 
competencies in locating emergency equipment include identifying opportunities for recurrent training for our learners. Additional opportunities exist for assessment of retention of previous training within pre-existing courses in the curriculum, such as orientation sessions occurring at least annually. Furthermore, based on our observations, it is logical to infer that additional training of faculty and staff may also be beneficial.

\section{5 | CONCLUSION}

In conclusion, our results support the incorporation of targeted handson training for dental students in the pre-doctoral curriculum on the location of medical emergency equipment in a clinical setting. As previously described, of the 138 students tested, only $10.14 \%$ of the students could locate seven of the nine items when compared to $100 \%$ in the novel group. Only $5.07 \%$ of students in the traditional group could locate all items initially, compared with $72.22 \%$ students in the novel group. Therefore, it is evident that the physical hands-on training is an effective educational tool.

\section{ACKNOWLEDGEMENTS}

The authors would like to extend sincere thanks to Dr. Mark Fitzgerald, Dr. Stephen Stefanac, Dr. Carol-Anne Murdoch Kinch and Dr. Russell Taichman of the University of Michigan School of Dentistry; Dr. David Sarment, President of Xoran Technologies; and Mr. Mark MacEachern, Informationist, Taubman Health Sciences Library, for their assistance with this study. We sincerely thank Dr. DM Rooney, University of Michigan School of Medicine, for her assistance with statistical analysis and interpretation of data.

\section{CONFLICTS OF INTEREST}

All authors declare that there are no conflicts of interest in relation to this study.

\section{DISCLOSURE}

All authors do not have any financial, economic or professional interests to disclose that may have influenced the design, execution or presentation of this scholarly work.

\section{REFERENCES}

1. Clark MS, Wall BE, Tholstrom TC, Christensen EH, Payne BC. A twenty-year follow-up survey of medical emergency education in U.S. dental schools. J Dent Educ. 2006;70:1316-1319.

2. Wald DA, Wang A, Carroll G, Trager J, Cripe J, Curtis M. An officebased emergencies course for third-year dental students. J Dent Educ. 2013;77:1033-1041.

3. Becker DE. Emergency drug kits: pharmacological and technical considerations. Anesth Prog. 2014;61:171-179.
4. Jevon P. Updated posters to help manage medical emergencies in the dental practice. Br Dent J. 2015;219:227-229.

5. Reznek M, Smith-Coggins R, Howard S, et al. Emergency medicine crisis resource management (EMCRM): pilot study of a simulation-based crisis management course for emergency medicine. Acad Emerg Med. 2003;10:386-389.

6. Scalese RJ, Obeso VT, Issenberg SB. Simulation technology for skills training and competency assessment in medical education. J Gen Intern Med. 2008;23:46-49.

7. Lundberg PW, Korndorffer JRJ. Using simulation to improve systems. Surg Clin North Am. 2015;95:885-892.

8. Pinsky HM, Taichman RS, Sarment DP. Adaptation of airline crew resource management principles to dentistry. J Am Dent Assoc. 2010;141:1010-1018.

9. Brooks-Buza H, Fernandez R, Stenger JP. The use of in situ simulation to evaluate teamwork and system organization during a pediatric dental clinic emergency. Simul Healthc. 2011;6:101-108.

10. Kolb DA. Experiential learning: experience as the source of learning and development. J Organ Behav [Internet]. 1984;8:19-38. http://learn ingfromexperience.com/media/2010/08/process-of-experientiallearning.pdf. Accessed August 24, 2015.

11. CODA Accreditation Document [Internet]. http://www.ada.org/ / media/CODA/Files/predoc.ashx. Accessed September 25, 2015.

12. Ericsson KA. An expert-performance perspective of research on medical expertise: the study of clinical performance. Med Educ. 2007:41:1124-1130.

13. Le TT, Scheller EL, Pinsky HM, Stefanac SJ, Taichman RS. Ability of dental students to deliver oxygen in a medical emergency. J Dent Educ. 2009;73:499-508.

14. Buchanan JA. Use of simulation technology in dental education. J Dent Educ. 2001;65:1225-1231.

15. Emery RW, Guttenberg SA. Management priorities and treatment strategies for medical emergencies in the dental office. Dent Clin North Am. 1999;43:401-419, v.

16. Rosenberg M. Preparing for medical emergencies: the essential drugs and equipment for the dental office. J Am Dent Assoc. 2010;141(Suppl):14S-19S.

17. Saha M, Saha S, Khandelwal A, Punjabi H. Emergency preparedness: a survey of dental practitioners in indore. J Indian Dent Assoc. 2011;5:1193-1195.

18. Malamed SF. Medical Emergencies in the Dental Office. 7th edn. Mosby: Elsevier; 2015. 576 p.

19. Malamed SF. Back to basics: emergency medicine in dentistry. J Calif Dent Assoc. 1997;25:285-286, 288-94.

20. McEvoy MD, Hand WR, Furse CM, et al. Validity and reliability assessment of detailed scoring checklists for use during perioperative emergency simulation training. Simul Healthc. 2014;9:295-303.

21. Al-Sebaei M, Alkayyal M, Alsulimani A, Alsulaimani O, Habib W. The preparedness of private dental offices and polyclinics for medical emergencies. a survey in Western Saudi Arabia. Saudi Med J. 2015;36:335-340.

22. Kumarswami S, Tiwari A, Parmar M, Shukla M, Bhatt A, Patel M. Evaluation of preparedness for medical emergencies at dental offices: a survey. J Int Soc Prev Community Dent. 2015;5:47-51.

23. Muller MP, Hansel M, Stehr SN, Weber S, Koch T. A state-wide survey of medical emergency management in dental practices: incidence of emergencies and training experience. Emerg Med J. 2008;25: 296-300.

24. Chapman PJ. Medical emergencies in dental practice and choice of emergency drugs and equipment: a survey of Australian dentists. Aust Dent J. 1997;42:103-108.

25. Broadbent JM, Thomson WM. The readiness of New Zealand general dental practitioners for medical emergencies. N Z Dent $J$. 2001;97:82-86 
26. Fast TB, Martin MD, Ellis TM. Emergency preparedness: a survey of dental practitioners. J Am Dent Assoc. 1986;112:499-501.

27. Gupta T, Aradhya MRS, Nagaraj A. Preparedness for management of medical emergencies among dentists in Udupi and Mangalore, India. J Contemp Dent Pract. 2008;9:92-99.

28. Atherton GJ, Pemberton MN, Thornhill MH. Medical emergencies: the experience of staff of a UK dental teaching hospital. Br Dent J. 2000;188:320-324.

29. Stafuzza T, Carrara C, Oliveira F, Santos C, Oliveira T. Evaluation of the Dentists' knowledge on medical urgency and emergency. Braz Oral Res. 2014;28:1-5.

30. Atherton GJ, McCaul JA, Williams SA. Medical emergencies in general dental practice in Great Britain. Part 2: drugs and equipment possessed by GDPs and used in the management of emergencies. Br Dent J. 1999;186:125-130.

31. Miller GE. The assessment of clinical skills/competence/performance. Acad Med. 1990;65:S63-S67.

32. Merrill MD. First principles of instruction. Educ Technol Res Dev. 2002;50:43-59.
33. Wecksell M, Vick A. The use of a scavenger hunt in CA-1 orientation to facilitate rapid identification and location of anesthetic equipment. MedEdPORTAL Publications. 2013;9:9506 https://doi.org/10.15766/ mep_2374-8265.9506. Accessed June 5, 2015.

34. Stross JK. Maintaining competency in advanced cardiac life support skills. JAMA. 1983;249:3339-3341.

35. Small SD, Wuerz RC, Simon R, Shapiro N, Conn A, Setnik G. Demonstration of high-fidelity simulation team training for emergency medicine. Acad Emerg Med. 1999;6:312-323.

How to cite this article: Pinsky HM, Le JM, Sweier DG, and Marti K. Dental students' ability to locate emergency equipment-lessons learned from aviation. Eur J Dent Educ, 2018;22:e19-e25. https://doi.org/10.1111/eje.12251 